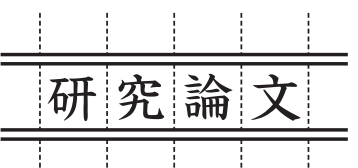

\title{
Prediction of the Period of Swirl Motion Appearing in Gas Injection Processes at High Temperatures
}

\author{
Akira HIRATSUKA*, Ryoji TSUJINO**, Yasushi SASAKI***, Kazuyoshi NISHIHARA**** and Manabu IGUCHI*****
}

(Received May 14, 2007)

\begin{abstract}
A swirl motion induced by gas injection through a bottom nozzle has high mixing ability and useful for the mixing of a molten steel bath in the refining processes. As one of the fundamental characteristics of the swirl motion, the period is mentioned in this study. A semi-empirical equation is proposed for the period of swirl motion by referring to the rotary sloshing appearing in a cylindrical vessel oscillated in the vertical or the horizontal direction.
\end{abstract}

Key Words: steel refining, high-temperature process, environmental problem, agitation, molten steel jet

\section{INTRODUCTION}

Bottom gas injection processes are extensively used in the steelmaking industries ${ }^{1-3)}$. Bubbles generated successively at the nozzle exit in a bath rise upward due to the buoyancy force acting on themselves. The bubble dispersion region thus formed above the nozzle is called the bubbling jet. The bubbles do not always rise straight upward. Under specific conditions the bubbling jet swirls around the vessel axis like the well-known rotary sloshing ${ }^{4,5)}$. The bath would be highly mixed in the presence of the swirl motion of the bubbling jet ${ }^{6}$.

The present authors are trying to develop a novel process using such a swirl motion to save energy and hence to decrease $\mathrm{CO}_{2}$ gas emission. The purpose of this study is to understand the period of swirl motion induced by gas injection through a centered bottom nozzle.

Two kinds of swirl motions are known to be induced in a bath contained in a cylindrical vessel by injecting gas into the bath ${ }^{5)}$, as shown in Fig.1.

The shallow-water wave type and deep-water wave type swirl motions appear depending on the aspect ratio of the bath. The critical aspect ratio is approximately 0.3 . The latter has a high mixing ability, and accordingly, it finds wide applications such as snow melting ${ }^{7)}$ and wastewater treatments ${ }^{8)}$.

As mentioned above, the swirl motions are very similar to the rotary sloshing, which is induced in a bath contained in a cylindrical vessel subjected to external oscillations in the horizontal or the vertical direction. The period of swirl motion was found to be approximated by an analytical equation derived for the period of rotary sloshing ${ }^{5}$. However, a clear difference can be seen between the period of swirl motion and that of rotary sloshing, although the difference is small. In this study discussion was given on the causes of this difference and a semi-empirical equation was proposed.

\section{FLOW FIELD IN THE PRESENCE OF SWIRL MOTION OF BUBBLING JET}

Previous studies on the swirl motion of a bubbling jet ${ }^{9)}$ revealed

\section{Occurrence condition of bottom blown swirling bubbling jet}

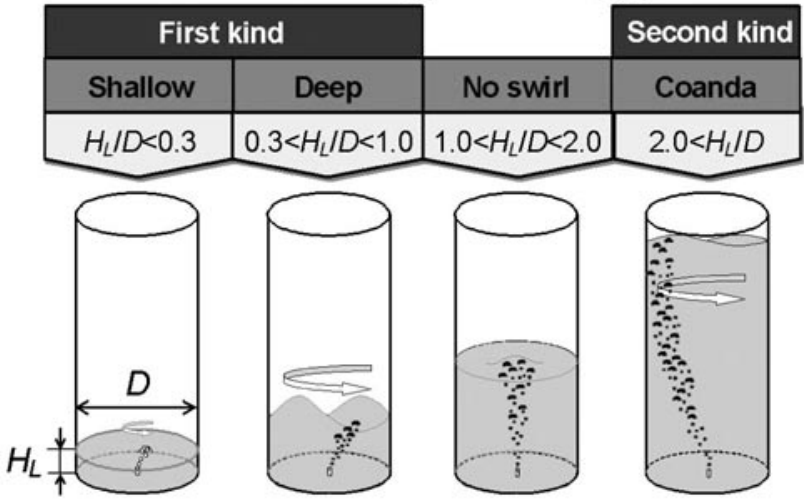

Fig.1 Swirl motions induced by bottom gas injection

\footnotetext{
* Faculty of Engineering, Osaka Sangyo University (3-1-1 Nakagaito, Daito, Osaka, 574-8530)

* * Faculty of Engineering, Setsunan University (17-8 Ikeda-Nakamachi, Neyagawa, 572-8508)

*** Graduate School of Engineering, Tohoku University (2 Aza-aoba, Aramaki, Aoba-ku, Sendai, 980-8579)

**** Graduate School of Engineering, Osaka Elctro-Communication University (18-8, Hatsu-cho, Neyagawa, 572-8530)

$* * * * *$ Graduate School of Engineering, Hokkaido University (North 13, West 8, Kita-ku, Sapporo, 060-8628)
} 
that when the bubbling jet rotates, for example, clockwise, the liquid outside the bubbling jet rotates counterclockwise so as to satisfy the conservation law of angular momentum. When the aspect ratio of the bath, $H_{L} / D$, is relatively small, the upper part of the liquid rotating counterclockwise reaches the bath surface. This motion can be clearly seen by putting small amount of silicone oil on the water bath surface. The region where the liquid moves counterclockwise on the bath surface becomes narrow as the aspect ratio, $H_{L} / D$, increases.

One more interesting feature of the flow field mentioned here is that bubbles of many sizes are contained in the bath. Large bubbles are successively generated at the nozzle exit. These bubbles are disintegrated into smaller bubbles having diameters over a wide range. In addition, many small bubbles are entrapped into the bath from the bath surface near the side wall of the vessel due to violent wave motions.

A total volume, $V_{g}$, of the large bubbles rising from the nozzle towards the bath surface can be predicted, as the bubble rising velocity, $u_{B}$, is known ${ }^{3}$.

$$
V_{g}=Q_{g} H_{L} / u_{B}
$$

where $Q_{g}$ is the gas flow rate and $H_{L}$ is the initial bath depth before gas injection. In the real situation the bath depth becomes greater than $H_{L}$ due to the existence of bubbles in the bath. This increment is neglected in this study because the total volume of gas, $V_{g}$, is much smaller than the volume of liquid, $V_{L}$, in the bath and the effect of bath depth on the period of swirl motion of the deep-water wave type is very small. It is difficult at the present stage to precisely predict the total volume of small bubbles entrapped from the bath surface into the bath. The small bubbles therefore are not mentioned in the following analysis.

The apparent density, $\rho_{a p p}$, of the mixture of liquid and gas in the bath is expressed by

$$
\rho_{\text {app }}=\left(\rho_{g} V_{g}+\rho_{L} V_{L}\right) /\left(V_{g}+V_{L}\right)
$$

where $\rho_{g}$ is the density of gas and $\rho_{L}$ is the density of liquid. By introducing an apparent acceleration due to gravity, $g_{a p p}$, the balance of forces acting on the bath is expressed by

$$
\rho_{g} V_{g} g+\rho_{L} V_{L} g-\rho_{L} V_{g} g=\rho_{a p p}\left(V_{g}+V_{L}\right) g_{a p p}
$$

where $g$ is the acceleration due to gravity.

Combination of Eqs.(2) and (3) yields

$$
g_{\text {app }}=\left[1-\rho_{L} V_{g} /\left(\rho_{g} V_{g}+\rho_{L} V_{L}\right)\right] g
$$

As $\rho_{g} V_{g}$ is much smaller than $\rho_{L} V_{L}$, Eq.(4) reduces to

$$
g_{\text {app }}=\left(1-V_{g} / V_{L}\right) g
$$

\section{EXPERIMENTAL APPARATUS}

Figure 2 shows a schematic diagram of the experimental apparatus. The cylindrical test vessel made of transparent acrylic resin had an inner diameter, $D$, of $80 \mathrm{~mm}$ to $390 \mathrm{~mm}$. The inner diameter of the nozzle, $d_{n}$, was varied from $1.0 \mathrm{~mm}$ to

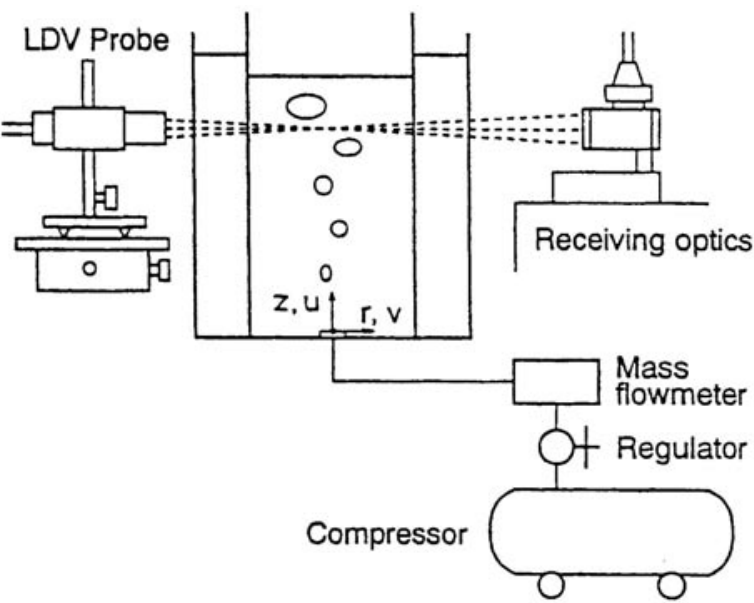

Fig.2 Experimental apparatus

\section{Rotary sloshing}

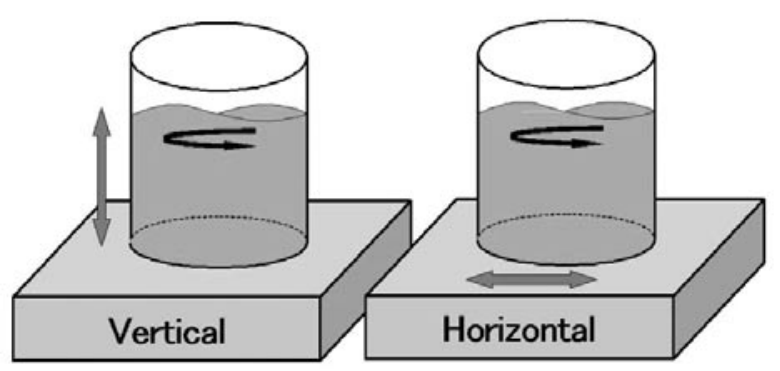

Fig.3 Rotary sloshing in a cylindrical vessel

$5.0 \mathrm{~mm}$. Water and mercury were used as the working fluids. The kinematic viscosity of water is nearly equal to that of molten steel. Air was injected into the test vessel with a compressor. The period of swirl motion was measured with a high-speed video camera and by eye inspection. The experimental results obtained with an LDV will be reported elsewhere.

\section{DERIVATION OF EQUATION FOR THE PERIOD OF SWIRL MOTION}

\subsection{PERIOD OF ROTARY SLOSHING}

Figure 3 shows a schematic illustration of the rotary sloshing appearing in a vessel containing partially filled liquid in it. Much information is available on the rotary sloshing. The period of the lowest mode of the rotary sloshing is expressed by

$$
T_{s}=2 \pi\left[(3.68 g / D) \tanh \left(3.68 H_{L} / D\right)\right]^{-1 / 2}
$$

A semi-empirical equation will be derived for the period of swirl motion of the deep-water wave type by referring to Eq.(6).

\subsection{PERIOD OF SWIRL MOTION OF BUBBLING JET}




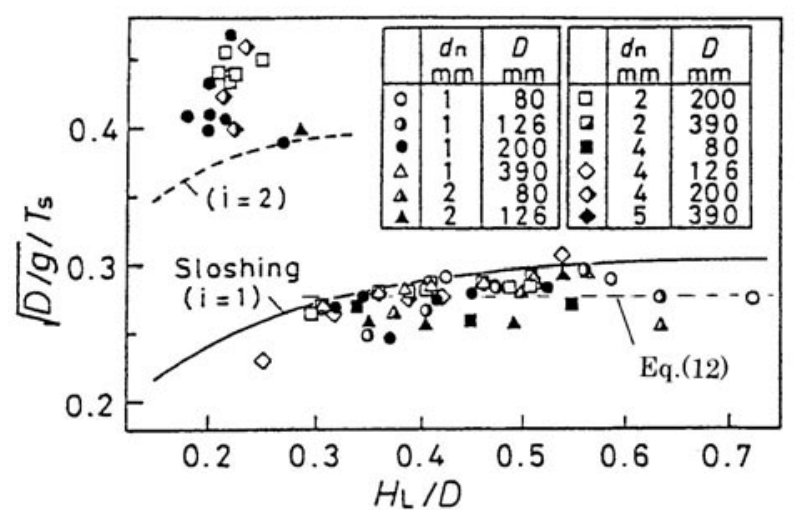

Fig.4 Comparison of measured values of the period of swirl motion in water bath with Eq.(12) and period for rotary sloshing

Figures 4 and 5 compare the measured values of the period of swirl motion with Eq.(6) for centered gas injection through a bottom nozzle ${ }^{10)}$. Equation (6) was denoted by a solid line in each figure. The deep-water wave type swirl motion appeared for an aspect ratio between about 0.3 and about unity. The difference between the measured values and E.(6) is small, but a definite difference can be seen. The value calculated from Eq.(6) decreased with a decrease in the aspect ratio, while the measured value remained almost unchanged in the region between $H_{L} / D$ $=0.3$ and 1.0 . We assume that such a difference is caused by the two fluid flow phenomena mentioned in the preceding section.

Equation (6) is modified as follows:

$T_{s}=2 \pi\left[\left(3.68 g_{\text {app }} / D_{\text {app }}\right) \tanh \left(3.68 H_{\text {Lapp }} / D_{\text {app }}\right)\right]^{-1 / 2}$

As mentioned earlier, it is difficult to precisely evaluate the total volume of bubbles existing in the bath, we further assume that $g_{\text {app }}$ is constant under the present experimental conditions and $H_{\text {Lapp }}$ is equal to $H_{L}$. Under these assumptions Eq.(7) reduces to

$$
1 / T_{s}=1 /(2 \pi)\left[\left(3.68 g_{\text {app }} / D_{\text {app }}\right) \tanh \left(3.68 H_{L} / D_{\text {app }}\right)\right]^{1 / 2}
$$

Multiplying the both sides of Eq. (8) by $(D / g)^{1 / 2}$ yields

$$
\begin{aligned}
(D / g)^{1 / 2} / T_{s}= & {[1 /(2 \pi)]\left[\left(g_{\text {app }} / g\right)\left(D / D_{\text {app }}\right)\right]^{1 / 2} } \\
& \times\left[3.68 \tanh \left\{3.68\left(H_{L} / D\right)\left(D / D_{\text {app }}\right)\right\}\right]^{1 / 2}
\end{aligned}
$$

In the following section expressions for $g_{a p p} / g$ and $D / D_{a p p}$ will be determined based on the experimental results of the present authors shown in Figs.4 and 5. It is reasonable to assume that $D / D_{a p p}=1.0$ for $H_{L} / D=1.0$, and accordingly, we have

$$
(D / g)^{1 / 2} / T_{s}=0.28 \quad\left(H_{L} / D=1.0\right)
$$

Substitution of Eq.(10) into Eq.(9) gives

$$
g_{\text {app }} / g=(0.918)^{2}
$$

Combination of Eq.(11) and Eq.(9) yields

$$
\begin{aligned}
(D / g)^{1 / 2} / T_{s}= & {[0.918 /(2 \pi)]\left(D / D_{\text {app }}\right)^{1 / 2} } \\
& \times\left[3.68 \tanh \left\{3.68\left(H_{L} / D\right)\left(D / D_{\text {app }}\right)\right\}\right]^{1 / 2}
\end{aligned}
$$

The measured values of $(D / g)^{1 / 2} / T_{s}$ are assumed to be nearly constant in the region from $H_{L} / D$ of about 0.3 to about unity.

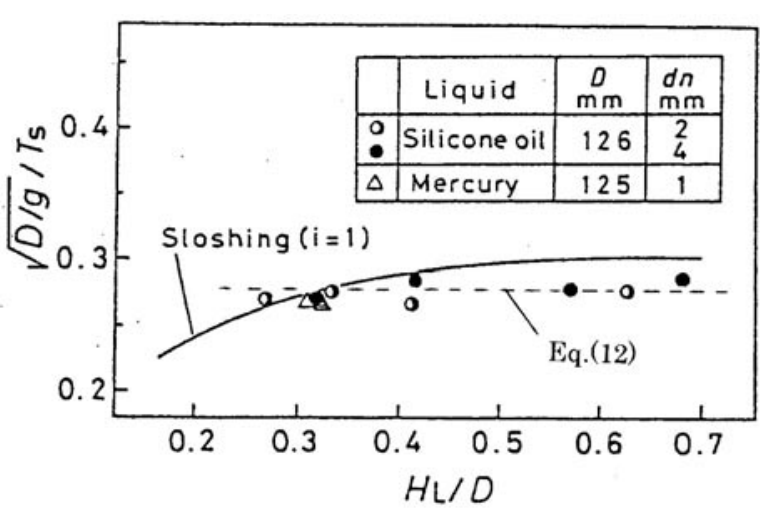

Fig.5 Comparison of measured values of the period of swirl motion in silicone and mercury baths with Eq.(12) and period for rotary sloshing

Substitution of $(D / g)^{1 / 2} / T_{S}=0.28$ and $H_{L} / D=0.3$ into Eq.(12) gives $D / D_{\text {app }}=1.17$

As one of the simplest expressions, the following linear relationship was chosen for $D / D_{a p p}$.

$$
D / D_{\text {app }}=1.25-0.25 H_{L} / D
$$

The period of swirl motion of the deep-water wave type for any aspect ratio can be estimated by substituting Eq.(14) into Eq.(12). Equation (12) is adequate for predicting the period of swirl motion of the deep-water wave type, as shown in Fids.4 and $\mathbf{5}$.

\subsection{APPLICABILITY OF Eq.(12) TO SWIRL MOTION OF LIQUID JET}

The period of a swirl motion caused by liquid jet injection ${ }^{11)}$ is known to be favorably approximated by the period of rotary sloshing, Eq. $(6)^{12)}$. This is because the inertial force of the injected liquid is directly used for driving the liquid in the bath. Accordingly, the bath is much more strongly agitated by the liquid jet than by gas injection. This fact suggests that the apparent diameter in Eq.(12), $D_{a p p}$, is nearly the same as the vessel diameter $D\left(D / D_{a p p} \approx 1\right)$, regardless of the aspect ratio. In addition, the volume of bubbles contained in the bath is much smaller than that in the bath with gas injection. This fact suggests that $g_{a p p} / g \approx$ 1 in Eq.(12).

\section{CONCLUSIONS}

The following semi-empirical equation was proposed for the period of swirl motion of the deep-water wave type appearing in the baths with bottom gas injection.

$$
\begin{aligned}
& (D / g)^{1 / 2} / T_{s}=[0.918 /(2 \pi)]\left(D / D_{\text {app }}\right)^{1 / 2} \\
& \times\left[3.68 \tanh \left\{3.68\left(H_{L} / D\right)\left(D / D_{\text {app }}\right)\right\}\right]^{1 / 2}
\end{aligned}
$$

The diameter ratio in Eq.(12) is given by

$$
D / D_{\text {app }}=1.25-0.25 H_{L} / D
$$


Prediction of the Period of Swirl Motion Appearing in Gas Injection Processes at High Temperatures $($ HIRATSUKA $\cdot$ TSUJINO $\cdot$ SASAKI $\cdot$ NISHIHARA $\cdot$ IGUCHI)

\section{NOMENCLATURE}

D vessel diameter

$D_{\text {app }}$ apparent vessel diameter

$d_{n} \quad$ inner diameter of nozzle

g acceleration due to gravity

$\mathrm{g}_{\text {app }}$ apparent acceleration due to gravity

$H_{L} \quad$ bath depth

$Q_{g} \quad$ gas flow rate

$T_{s} \quad$ period of swirl motion

$u_{B} \quad$ bubble rising velocity

$V_{g} \quad$ total volume of bubbles in the bath

$V_{L} \quad$ volume of liquid in the bath

$\rho_{a p p} \quad$ apparent density

$\rho_{g} \quad$ density of gas

$\rho_{L} \quad$ density of liquid

\section{REFERENCES}

1. K. Mori and M. Sano, Tetsu-to-Hagane, 67(1981), 672.

2. D. Mazumdar and R. I. L. Guthrie, ISIJ Int., 35-1(1995), 1.

3. O. J. Ilegbusi, M. Iguchi, and W. Wahnsiedler. Mathematical and
Physical Modeling of Materials Processing Operations, Chapman \& Hall/CRC, (1999).

4. A. Kimura and H. Ohashi, Trans. Jpn. Mech. Soc. Eng., Pt.1, 44(1978), 3024.

5. Jpn. Soc. Fluid Dynamics, Handbook of Fluid Dynamics, Marzen(1987)

6. O. J. Ilegbusi, M. Iguchi, and W. Wahnsiedler. Mathematical and Physical Modeling of Materials Processing Operations, Chapman \& Hall/CRC, (1999), 380.

7. M. Iguchi, Y. Sasaki, K. Sasaki and J. Yoshida, Jpn. J. Multiphase Flow, 16-3(2002), 232.

8. M. Shitara, M. Iguchi, K. Takano, T. Tamamori, H. Shitara and T. Maruyama, Materials Transactions, 44-12(2003), 2456.

9. M. Iguchi, T. Kondoh, T. Uemura, F. Yamamoto and Z. Morita, Experiments in Fluids, 16(1994), 255.

10. M. Iguchi, S. Hosohara, T. Koga, R. Yamaguchi and Z. Morita, ISIJ Int., 33-10(1993), 729.

11. H. Madarame, K. Okamoto and M. Iida: J. Fluids Structure, 16 (2002), 417.

12. J. Yoshida, D. Iguchi, and M. Iguchi: ISIJ Int., 43 (2003), 1890. 